\title{
Risk Factors Associated With Symptomatic Radiation Pneumonitis After Stereotactic Body Radiation Therapy for Stage I Non-Small Cell Lung Cancer
}

Technology in Cancer Research \& Treatment

2017, Vol. 16(3) 316-320

(C) The Author(s) 2016

Reprints and permission:

sagepub.com/journalsPermissions.nav DOI: I0.1 I77/I5330346/666/665 journals.sagepub.com/home/tct

@SAGE

\author{
Shiming Shi, BSc', Zhaochong Zeng, MD, PhD', Luxi Ye, BSc', \\ Yan Huang, BSc', and Jian He, MD, PhD'
}

\begin{abstract}
Radiation pneumonitis is the most frequent acute pulmonary toxicity following stereotactic body radiation therapy for lung cancer. Here, we investigate clinical and dosimetric factors associated with symptomatic radiation pneumonitis in patients with stage I non-small cell lung cancer treated with stereotactic body radiation therapy. A total of 67 patients with stage I non-small cell lung cancer who received stereotactic body radiation therapy at our institution were enrolled, and their clinicopathological parameters and dosimetric parameters were recorded and analyzed. The median follow-up period was 26.4 months (range: 7-48 months). In univariate analysis, tumor size $(P=.04 \mathrm{I})$, mean lung dose $(P=.028), \mathrm{V} 2.5(P=.024), \mathrm{V} 5(P=.014), \mathrm{VI0}(P=.004)$, V20 $(P=.024), \mathrm{V} 30(P=.020), \mathrm{V} 40(P=.040)$, and V50 $(P=0.040)$ were associated with symptomatic radiation pneumonitis. In multivariable logistic regression analysis, VIO $(P=.049)$ was significantly associated with symptomatic radiation pneumonitis. In conclusion, this study found that tumor size, mean lung dose, and V2.5 to V50 were risk factors markedly associated with symptomatic radiation pneumonitis. Our data suggested that lung VIO was the most significant factor, and optimizing lung $\mathrm{V} I 0$ may reduce the risk of symptomatic radiation pneumonitis. For both central and peripheral stage I lung cancer, rate of radiation pneumonitis $\geq$ grade 2 was low after stereotactic body radiation therapy with appropriate fraction dose.
\end{abstract}

\section{Keywords}

non-small cell lung cancer, stereotactic body radiation therapy, radiation pneumonitis, risk factor, helical tomotherapy

\begin{abstract}
Abbreviations
ADLs, activities of daily living; BED, biological effective dose; COPD, chronic obstructive pulmonary disease; CT, computed tomography; 4D-CT, 4-dimensional CT; FDG, fluorodeoxyglucose; GTV, gross tumor volume; ITV, internal target volume; KPS, Karnofsky performance score; MLD, mean lung dose; NSCLC, non-small cell lung cancer; PET, positron-emission tomography; PTV, planning target volume; RP, radiation pneumonitis; SBRT, stereotactic body radiotherapy; Vx, volume of both lungs minus the gross tumor volume receiving $x$ Gy.
\end{abstract}

Received: October II, 2015; Revised: May 22, 2016; Accepted: July I, 2016.

\section{Introduction}

Lung cancer was the most frequently diagnosed cancer and the leading cause of cancer death among males in 2012. For females, lung cancer was the leading cause of cancer death in developed countries and the second leading cause of cancer death in less developed countries. ${ }^{1}$ Pulmonary lobectomy associated with mediastinal lymph node dissection is the standard treatment for early-stage lung cancer, with excellent local control rate of more than $90 \%$ and 5 -year survival of more than $50 \%{ }^{2}$ However, many elderly patients cannot tolerate surgery because of poor pulmonary function or severe complications. Radiotherapy is a reasonable option for lung cancer treatment among those who are not eligible for surgery.

Stereotactic body radiation therapy is a relatively new procedure for treating lung cancer that uses high doses of radiation

\footnotetext{
' Department of Radiation Oncology, Zhongshan Hospital, Fudan University, Shanghai, China
}

\section{Corresponding Author:}

Jian He, MD, PhD, Department of Radiation Oncology, Zhongshan Hospital, Fudan University, Shanghai 200032, China.

Email: hejian62@।63.com 
delivered to a precise target. By using special positioning and implanted markers in the body, radiation oncologists are able to deliver a much higher dose of radiation to gross tumor volume (GTV) than traditional radiation therapy, while sparing healthy tissue. It could shorten the treatment course and elevate radiation dose compared with traditional regimens. Stereotactic body radiation therapy has been an excellent treatment option for early-stage lung cancer.

Radiation pneumonitis (RP) is one of the most common toxicities of stereotactic body radiotherapy (SBRT), which may reduce the patients' quality of life. ${ }^{3}$ Therefore, being able to predict the risk of RP after SBRT is of great importance in treatment planning. In this study, we retrospectively gathered clinicopathological parameters and dosimetric parameters from the dose-volume histogram curves of the individual treatment plans. We searched for risk factors associated with RP after SBRT for early-stage lung cancer, so as to further optimize the treatment of SBRT for non-small cell lung cancer (NSCLC).

\section{Methods and Materials}

\section{Patients}

A total of 67 patients with stage I NSCLC who received SBRT at our institution were enrolled. These patients were medically inoperable or refused to undergo surgery. Lung cancer stage was classified according to the seventh edition of TNM Classification of Malignant Tumours by American Joint Committee on Cancer using computed tomography (CT) of the chest and upper abdomen, bone scintigraphy, and brain magnetic resonance imaging. Sixty patients were diagnosed by pathology and 7 patients were diagnosed by multidisciplinary team, composed of doctors from radiology, pulmonary, radiotherapy, and thoracic surgery departments. Clinical diagnosis of lung cancer depended on consecutive increase in tumor size in CT or pathological uptake of ${ }^{18} \mathrm{~F}$-fluorodeoxyglucose (FDG) in the lesion in positron emission tomography (PET) scan. Staging of negative $\mathrm{N}$ and $\mathrm{M}$ status was based on contrast-enhanced $\mathrm{CT}$ scans or PET-CT.

Their following clinicopathological parameters and dosimetric parameters were recorded and analyzed-age; gender; Karnofsky performance score (KPS); pathological types, tumor position and stage; chronic obstructive pulmonary disease (COPD); mean lung dose (MLD); planning target volume (PTV); and V2.5, V5, V10, V20, V30, V40, V50.

The study was approved by ethics committee of the Zhongshan Hospital, and protocols of institutional review board were followed when informed consent was obtained from each patient.

\section{Treatment Planning}

All patients were immobilized by body fixation and simulated by respiration-correlated 4-dimensional CT (4D-CT) with slice thickness of $3 \mathrm{~mm}$. Breathing mobility was derived from dynamic 4D-CT scanning and the Real-Time Position
Management system. The primary tumor in the enhanced CT or FDG-PET/CT was delineated as GTV, and no additional margin was added for the generation of the clinical target volume. Internal target volume (ITV) was generated including the extension of GTVs at all phases of the respiratory cycle on the 4D-CT scanning. The PTV extended a margin of $0.3 \mathrm{~cm}$ from the ITV to account for compensation errors of the tumor position and changes in the tumor motion caused by breathing. The ${ }^{18}$ FDG-PET-guided radiotherapy planning was optional. The tumor region was scanned daily, and the patient was repositioned based on image-guided radiotherapy. Target delineation was performed in the Pinnacle treatment planning system (Philips Medical Systems, Bothell, Washington). Afterward, each patient's planning CT scans and the contours were transferred into the helical tomotherapy Hi-Art II planning system (TomoTherapy, Madison, Wisconsin) for treatment planning. Patients with peripherally located lesions (ie, those located $>2 \mathrm{~cm}$ in any direction from the proximal bronchial tree, major vessels, esophagus, heart, tracheal, vertebral body, pericardium, mediastinal pleural, and brachial plexus) received a total radiation dose of $50 \mathrm{~Gy}$ in five $10 \mathrm{~Gy}$ fractions, and the biological effective dose (BED) was $100 \mathrm{~Gy} .{ }^{4}$ For central lesions (ie, those within $2 \mathrm{~cm}$ of these structures), 60 Gy in ten 6 Gy fractions was used, and the BED was $96 \mathrm{~Gy}$. The prescription isodose surface had to be $\geq 60 \%$ and $<90 \%$ of the maximum dose. Adequate target coverage was achieved when $95 \%$ of the PTV was covered by the assigned total dose and when $99 \%$ of the PTV received $\geq 90 \%$ of the prescription dose. Dose constraints for organs at risk were maintained on the basis of the criteria described by the National Comprehensive Cancer Network (NCCN) Clinical Practice Guidelines in Oncology: NonSmall Cell Lung Cancer (version 4.2014).

\section{Radiation Pneumonitis Grading and Follow-Up}

Radiation pneumonitis was graded according to the Common Terminology Criteria for Adverse Events version 4.0 for pneumonitis, which consisted of the following grades - grade 1, asymptomatic with radiographic findings only; grade 2, symptomatic and not interfering with the activities of daily living (ADLs); grade 3, symptomatic, interfering with the ADLs and $\mathrm{O}_{2}$ indicated; and grade 4, life-threatening, ventilatory support indicated. Symptomatic RP (grade $\geq 2$ ) was analyzed in this study. The patients were divided into 2 groups-RP group ( $\geq 2$ grade RP) and no-RP group ( 0 and 1 grade $R P$ ).

Routine follow-up consisted of visits after 3, 6, 12, 18, and 24 months and yearly thereafter. At each follow-up visit, new symptoms were recorded, and a diagnostic CT scan was performed. The PET-CT was optional and mainly performed when tumor relapse was suspected.

\section{Statistical Analysis}

Chi-square test and Student $t$ test were used to determine whether differences concerning clinical and dosimetric factors existed between the patients with and without symptomatic RP. 
Table 1. Clinicopathological Characteristics.

\begin{tabular}{|c|c|c|c|}
\hline & $\begin{array}{l}\text { No-RP Group } \\
(\mathrm{n}=59), \mathrm{n}(\%)\end{array}$ & $\begin{array}{c}\text { RP Group } \\
(\mathrm{n}=8), \mathrm{n}(\%)\end{array}$ & $P$ \\
\hline Age, years & & & $.382^{\mathrm{a}}$ \\
\hline Median & 72.95 & 69.63 & \\
\hline Range & $49-88$ & $48-80$ & \\
\hline Gender & & & .627 \\
\hline Male & 49 & 6 & \\
\hline Female & 10 & 2 & \\
\hline KPS & & & .482 \\
\hline$\geq 80$ & 55 & 7 & \\
\hline$<80$ & 4 & 1 & \\
\hline Histologic type & & & .973 \\
\hline Squamous cell carcinoma & 24 & 3 & \\
\hline Adenocarcinoma & 29 & 4 & \\
\hline No biopsy & 6 & 1 & \\
\hline Tumor size & & & .041 \\
\hline$\leq 2 \mathrm{~cm}$ & 30 & 1 & \\
\hline$>2 \mathrm{~cm}$ & 29 & 7 & \\
\hline Location & & & .450 \\
\hline Central & 32 & 6 & \\
\hline Peripheral & 27 & 2 & \\
\hline Lobe of lung & & & .872 \\
\hline LUL & 21 & 3 & \\
\hline LLL & 10 & 2 & \\
\hline RUL & 12 & 2 & \\
\hline RML & 6 & 0 & \\
\hline RLL & 10 & 1 & \\
\hline COPD & & & .138 \\
\hline Yes & 20 & 5 & \\
\hline No & 39 & 3 & \\
\hline
\end{tabular}

Abbreviations: COPD, chronic obstructive pulmonary disease; LLL, left lower lobe; LUL, left upper lobe; KPS, Karnofsky performance score; RLL, right lower lobe; RML, right middle lobe; RP, radiation pneumonitis; RUL, right upper lobe.

${ }^{a} P$ value from Student $t$ test; $\chi^{2}$ test or Fisher exact test for all other analyses.

Categorical variables were compared using 2-sided Pearson $\chi^{2}$ test, and for small group samples, Fisher exact test was applied. Continuous variables were compared using unpaired Student $t$ test. The variables that were significantly different $(P<.05)$ between the 2 groups by univariate analysis were then included in a multivariate analysis using a logistic regression test. The optimal cutoff values for V2.5 to V50 were determined using time-dependent receiver operating characteristic curve. This analysis was conducted in SPSS software, version 19.0 (SPSS Inc, Chicago, Illinois). Statistical significance was defined as $P<.05$.

\section{Results}

From June 2011 to August 2014, we treated 67 patients with stage I NSCLC using SBRT. All patients completed the treatment and had 6 or more months of follow-up regarding RP. The median follow-up period was 26.4 months (range: 7-48 months). The incidence of grade 1, 2, and 3 RP after SBRT was $29.9 \%$ (20 of 67 ), $10.4 \%$ ( 7 of 67 ), and $1.5 \%$ (1 of 67), respectively.
Table 2. Dosimetric Parameters.

\begin{tabular}{|c|c|c|c|}
\hline Parameter & $\begin{array}{c}\text { No-RP Group } \\
\text { (Median } \pm \text { SD) }\end{array}$ & $\begin{array}{c}\text { RP Group } \\
\text { (Median } \pm \text { SD) }\end{array}$ & $P$ \\
\hline PTV volume, $\mathrm{cm}^{3}$ & $35.1 \pm 30.6$ & $45.4 \pm 29.2$ & $.403^{\mathrm{a}}$ \\
\hline MLD, Gy & $1.19 \pm 1.10$ & $3.03 \pm 1.98$ & $.028^{\mathrm{a}}$ \\
\hline $\mathrm{V} 2.5, \%$ & $37.7 \pm 12.7$ & $46.1 \pm 18.4$ & .024 \\
\hline $\mathrm{V} 5, \%$ & $25.1 \pm 9.9$ & $36.1 \pm 15.5$ & .014 \\
\hline $\mathrm{V} 10, \%$ & $14.7 \pm 7.3$ & $27.6 \pm 13.8$ & .004 \\
\hline $\mathrm{V} 20, \%$ & $6.7 \pm 4.3$ & $13.2 \pm 8.4$ & .024 \\
\hline V30, \% & $3.5 \pm 2.4$ & $7.4 \pm 5.3$ & .020 \\
\hline $\mathrm{V} 40, \%$ & $1.9 \pm 1.4$ & $4.1 \pm 3.7$ & .040 \\
\hline V50, \% & $0.9 \pm 1.4$ & $2.3 \pm 2.5$ & .040 \\
\hline
\end{tabular}

Abbreviations: MLD, mean lung dose; PTV, planning target volume; RP, radiation pneumonitis; $\mathrm{SD}$, standard deviation; Vx, percentage of lung volume exceeding x Gy.

${ }^{a} P$ value from Student $t$ test; Mann-Whitney $U$ test for all other analyses.

Table 3. Multivariable Logistic Regression Analyses of Factors Associated With Symptomatic RP.

\begin{tabular}{ll}
\hline Parameter & $P$ \\
\hline Tumor size $(\leq 2 \mathrm{vs}>2 \mathrm{~cm})$ & .283 \\
V2.5, $\%$ & .114 \\
V5, \% & .691 \\
V10, \% & .049 \\
V20, \% & .073 \\
V30, \% & .336 \\
V40, \% & .265 \\
V50, \% & .109 \\
MLD, Gy & .091 \\
\hline
\end{tabular}

Abbreviations: MLD, mean lung dose; RP, radiation pneumonitis; Vx, percentage of lung volume exceeding $x$ Gy.

The clinicopathological characteristics analyzed are summarized in Table 1. No statistically significant difference was found for symptomatic RP development between the RP group and the no-RP group in terms of age, gender, KPS, pathological types, tumor position, and COPD. Tumor size $(P=.041)$ was analyzed as risk factor associated with symptomatic RP.

The dosimetric characteristics analyzed are summarized in Table 2. Univariate analysis suggested the following dosimetric factors were risk factors associated with symptomatic $\mathrm{RP}-\mathrm{V} 2.5(P=.024), \mathrm{V} 5(P=.014), \mathrm{V} 10(P=.004), \mathrm{V} 20$ $(P=.024), \mathrm{V} 30(P=.020), \mathrm{V} 40(P=.040), \mathrm{V} 50(P=.040)$, and MLD $(P=.028)$. When the patients were evaluated in terms of PTV volume, no statistically significant difference was found between the groups with and without symptomatic RP.

Subsequent multivariate analysis between the 2 groups was performed with the variables that showed univariate significance $(P<.05)$. As shown in Table 3 , the significant factor was V10 $(P=.049)$.

The optimal cutoff values for V2.5 to V50 were determined using time-dependent receiver operating characteristic curve. As shown in Table 4, the incidence of symptomatic RP was 
Table 4. The Optimal Cutoff Value for V2.5 to V50 to Protect From Symptomatic RP.

\begin{tabular}{|c|c|c|c|}
\hline & $\begin{array}{l}\text { No-RP Group } \\
(\mathrm{n}=59), \mathrm{n}(\%)\end{array}$ & $\begin{array}{c}\text { RP Group } \\
(\mathrm{n}=8), \mathrm{n}(\%)\end{array}$ & $P$ \\
\hline V2.5 & & & .001 \\
\hline$<49 \%$ & 51 & 3 & \\
\hline$\geq 49 \%$ & 8 & 5 & \\
\hline $\mathrm{V} 5^{-}$ & & & .000 \\
\hline$<33.5 \%$ & 54 & 3 & \\
\hline$\geq 33.5 \%$ & 5 & 5 & \\
\hline $\mathrm{V} 1 \overline{0}$ & & & .000 \\
\hline$<19 \%$ & 49 & 2 & \\
\hline$\geq 19 \%$ & 10 & 6 & \\
\hline $\mathrm{V} 2 \overline{0}$ & & & .000 \\
\hline$<16 \%$ & 57 & 3 & \\
\hline$\geq 16 \%$ & 2 & 5 & \\
\hline $\mathrm{V} 3 \overline{0}$ & & & .000 \\
\hline$<7.5 \%$ & 56 & 3 & \\
\hline$\geq 7.5 \%$ & 3 & 5 & \\
\hline $\mathrm{V} 4 \overline{0}$ & & & .001 \\
\hline$<3.5 \%$ & 54 & 4 & \\
\hline$\geq 3.5 \%$ & 5 & 4 & \\
\hline
\end{tabular}

Abbreviations: RP, radiation pneumonitis; Vx, percentage of lung volume exceeding x Gy.

significantly lower in the patients with a cutoff value of V2.5 $<49 \%$ versus $\geq 49 \%(P=.001)$; in the patients with a cutoff value of $\mathrm{V} 5<33.5 \%$ versus $\geq 33.5 \%(P=.001)$; in the patients with a cutoff value of V10<19\% versus $\geq 19 \%(P=.001)$; in the patients with a cutoff value of V20 $<16 \%$ versus $\geq 16 \%$ $(P<.001)$; in the patients with a cutoff value of $\mathrm{V} 30<7.5 \%$ versus $\geq 7.5 \%(P<.001)$; and in the patients with a cutoff value of $\mathrm{V} 40<3.5 \%$ versus $\geq 3.5 \%(P<.001)$.

\section{Discussion}

Radiation pneumonitis is the most frequent acute pulmonary toxicity following SBRT for lung cancer. Although most RP is grade 1 or 2 and either asymptomatic or manageable, severe cases can be seen and there is a risk for mortality..$^{5}$ The purpose of this study was to identify risk factors associated with symptomatic RP ( $\geq$ grade 2 ) following SBRT for stage I NSCLC.

The reported rates of symptomatic RP after SBRT range from $5.2 \%$ to $21 \% .^{6-12}$ In this study, the incidence of symptomatic RP after SBRT was $11.9 \%$, consisting of $7(10.4 \%)$ patients with grade 2 and $1(1.5 \%)$ patient with grade 3 , which is consistent with most previous reports.

Several clinical factors were investigated to predict the occurrence or severity of RP in our research. Our analysis suggested tumor size was the only clinicopathological parameter associated with symptomatic RP, similar to some previous studies that reported SBRT-related pulmonary toxicity correlated with large tumor volumes. ${ }^{6,11} \mathrm{We}$ found the proportion of patients with symptomatic RP was significantly lower among patients with tumor size $\leq 2 \mathrm{~cm}$ than those $>2 \mathrm{~cm}$. Other clinical factors reported to be predictive of RP in previous studies, including gender, ${ }^{7,8}$ combining with chronic lung disease $^{10}$ and tumor located in the middle or lower lobe. ${ }^{10}$ However, there was no significant association between these factors and symptomatic RP based on the present study.

The effect of dosimetric parameters was thought to be crucial for the development of RP. It is well accepted in radiation oncology that reducing the volume of normal lung irradiated is an effective technique for lowering morbidity. Several dosevolume parameters have been identified to be predictive of RP. Barriger et $\mathrm{al}^{13}$ found that both the total lung MLD and V20 correlated with RP, with a $4 \%$ incidence of RP observed if the MLD was $\leq 4$ Gy versus a $18 \%$ incidence if this value was $>4$ Gy. Chang et al $^{14}$ found the ipsilateral MLD to be the only significant factor on multivariate analysis, with an RP incidence of $93 \%$ if MLD $\geq 9.1$ Gy versus $7 \%$ if $<9.1 \mathrm{~Gy}$. Guckenberger et $\mathrm{al}^{11}$ found the MLD and V2.5 to V50 of the ipsilateral lung were correlated with the incidence of RP after pulmonary SBRT. Similarly, univariate analysis of our data suggested MLD and V2.5 to V50 are risk factors associated with symptomatic RP after SBRT therapy for stage I lung cancer, and V10 remained significant in multivariate analysis. The optimal cutoff value for V10 was $19 \%$, which was determined using time-dependent receiver operating characteristic curve. Within our population, the incidence of grade $\geq 2 \mathrm{RP}$ was found to be significantly lower in the patients with a cutoff value for $\mathrm{V} 10<19 \%$ versus $\geq 19 \%$ ( $P<.001)$. In our study, patients with $\mathrm{V} 10<19 \%$ had an RP risk of $3.9 \%$ ( 2 of 51 ), and those with $\mathrm{V} 10 \geq 19 \%$ had a significantly higher RP risk of $37.5 \%$ ( 6 of 16). Thus, V10 may be one of the most important factors for RP, and we can keep the value less than $19 \%$ to reduce the $R P$ incidence during radiation. Some previous studies concluded the symptomatic RP rate was significantly lower in the group with smaller PTV compared with the larger PTV group. ${ }^{6,15}$ However, there was no significant association between PTV and symptomatic RP based on the present study. As the number of patients is small, the accuracy of the results needs to be validated by more studies. Additionally, it has to be pointed out that the fractionation schedules utilized in our study were not the same as previous studies, and the heterogeneity of the dose prescriptions may also contribute to the difference in research results.

Respiratory motion is a nonnegligible source of uncertainty in radiotherapy. Intrafractional tumor motion is thought to increase the risk of geographic miss. At our institution, a 4D-CT simulation process is used to tackle with tumor motion. Four-dimensional CT can be adopted to evaluate the respiration motion of lung tumor accurately, and the PTV encompasses an internal tumor volume composed of the extension of GTVs at the all phases of the respiratory cycle. The 4D-CT-based PTV definition and radiotherapeutic planning can reduce the volume of PTV, increase the intratarget dose, and decrease the dose of normal tissue sequentially, reducing the risk of geographic miss. Efforts should be made to reduce the risk of RP using methods such as 4D-CT with respiratory gating or real-time tumor-tracking radiation therapy systems. ${ }^{16,17}$

The present study had several limitations. First, some previous studies suggested low pulmonary function was related to 
the occurrence of RP following SBRT, ${ }^{10,18}$ but pulmonary function tests were not gathered in our analyses because it was not routinely measured in our clinical practice. In addition, the number of symptomatic RP events was limited in the present study, and the heterogeneous sizes of the groups (59 patients vs 8 patients) may weaken the convincingness of our conclusions. Large-scale studies are warranted to substantiate and validate our results.

In conclusion, our study found that tumor size, MLD, and V2.5 to V50 were risk factors significantly associated with symptomatic RP. Our data suggested that lung V10 was the most significant factor and optimizing lung V10 may reduce the risk of symptomatic RP. For both central and peripheral stage I lung cancer, rate of RP $\geq$ grade 2 was low after SBRT with appropriate fraction dose.

\section{Authors' Note}

Shiming Shi and Zhaochong Zeng contributed equally to this work. Jian He and Zhaochong Zeng designed the overall study. Jian He treated most of the patients with lung cancer and drafted the manuscript. Shiming Shi collected the clinical data, performed the statistical analysis, and cowrote the paper. Luxi Ye and Yan Huang performed the follow-up. All authors read and approved the final manuscript.

\section{Declaration of Conflicting Interests}

The author(s) declared no potential conflicts of interest with respect to the research, authorship, and/or publication of this article.

\section{Funding}

The author(s) disclosed receipt of the following financial support for the research, authorship, and/or publication of this article: This work was supported by the Medical Guidance Program of the Science and Technology Commission of Shanghai Municipality (No 14411970100).

\section{References}

1. Torre LA, Bray F, Siegel RL, Ferlay J, Lortet-Tieulent J, Jemal A. Global cancer statistics, 2012. CA Cancer J Clin. 2015;65(2): 187-108. doi:10.3322/caac.21262.

2. Rami-Porta R, Crowley JJ, Goldstraw P. The revised TNM staging system for lung cancer. Ann Thorac Cardiovasc Surg. 2009; 15(1):4-9.

3. Ricardi U, Badellino S, Filippi AR. Stereotactic body radiotherapy for early stage lung cancer: history and updated role. Lung Cancer. 2015;90(3):388-396. doi:10.1016/j.lungcan. 2015.10.016.

4. Chang JY, Senan S, Paul MA, et al. Stereotactic ablative radiotherapy versus lobectomy for operable stage I non-small-cell lung cancer: a pooled analysis of two randomised trials. Lancet Oncol. 2015;16(6):630-637. doi:10.1016/S1470-2045(15)70168-3.

5. Yamashita H, Nakagawa K, Nakamura N, et al. Exceptionally high incidence of symptomatic grade 2-5 radiation pneumonitis after stereotactic radiation therapy for lung tumors. Radiat Oncol. 2007;2:21. doi:10.1186/1748-717X-2-21.

6. Baker R, Han G, Sarangkasiri S, et al. Clinical and dosimetric predictors of radiation pneumonitis in a large series of patients treated with stereotactic body radiation therapy to the lung. Int $J$ Radiat Oncol Biol Phys. 2013;85(1):190-195. doi:10.1016/j. ijrobp.2012.03.041.

7. Takeda A, Ohashi T, Kunieda E, et al. Comparison of clinical, tumour-related and dosimetric factors in grade 0 -1, grade 2 and grade 3 radiation pneumonitis after stereotactic body radiotherapy for lung tumours. Br J Radiol. 2012;85(1013):636-642. doi:10. 1259/bjr/71635286.

8. Robnett TJ, Machtay M, Vines EF, McKenna MG, Algazy KM, McKenna WG. Factors predicting severe radiation pneumonitis in patients receiving definitive chemoradiation for lung cancer. Int $J$ Radiat Oncol Biol Phys. 2000;48(1):89-94.

9. Hope AJ, Lindsay PE, El NI, et al. Modeling radiation pneumonitis risk with clinical, dosimetric, and spatial parameters. Int $J$ Radiat Oncol Biol Phys. 2006;65(1):112-124. doi:10.1016/j. ijrobp.2005.11.046.

10. Zhang XJ, Sun JG, Sun J, et al. Prediction of radiation pneumonitis in lung cancer patients: a systematic review. J Cancer Res Clin Oncol. 2012;138(12):2103-2116. doi:10.1007/s00432-0121284-1.

11. Guckenberger M, Baier K, Polat B, et al. Dose-response relationship for radiation-induced pneumonitis after pulmonary stereotactic body radiotherapy. Radiother Oncol. 2010;97(1):65-70. doi: 10.1016/j.radonc.2010.04.027.

12. Zhu $\mathrm{Z}, \mathrm{Fu} \mathrm{X}$. The radiation techniques of tomotherapy \& intensity-modulated radiation therapy applied to lung cancer. Transl Lung Cancer Res. 2015;4(3):265-274. doi:10.3978/j.issn. 2218-6751.2015.01.07.

13. Barriger RB, Forquer JA, Brabham JG, et al. A dose-volume analysis of radiation pneumonitis in non-small cell lung cancer patients treated with stereotactic body radiation therapy. Int $J$ Radiat Oncol Biol Phys. 2012;82(1):457-462. doi:10.1016/j. ijrobp.2010.08.056.

14. Chang JY, Liu H, Balter P, et al. Clinical outcome and predictors of survival and pneumonitis after stereotactic ablative radiotherapy for stage I non-small cell lung cancer. Radiat Oncol. 2012;7: 152. doi:10.1186/1748-717X-7-152.

15. Matsuo Y, Shibuya K, Nakamura M, et al. Dose-volume metrics associated with radiation pneumonitis after stereotactic body radiation therapy for lung cancer. Int J Radiat Oncol Biol Phys. 2012;83(4):e545-e549. doi:10.1016/j.ijrobp.2012.01.018.

16. Inoue $\mathrm{T}$, Katoh $\mathrm{N}$, Onimaru R, et al. Stereotactic body radiotherapy using gated radiotherapy with real-time tumor-tracking for stage I non-small cell lung cancer. Radiat Oncol. 2013;8:69. doi: 10.1186/1748-717X-8-69.

17. van der Voort VZN, van der Holt B, van Klaveren RJ, Pattynama P, Maat A, Nuyttens JJ. Stereotactic body radiotherapy using realtime tumor tracking in octogenarians with non-small cell lung cancer. Lung Cancer. 2010;69(3):296-301. doi:10.1016/j.lungcan.2009.12.008.

18. Inoue T, Shiomi H, Oh RJ. Stereotactic body radiotherapy for Stage I lung cancer with chronic obstructive pulmonary disease: special reference to survival and radiation-induced pneumonitis. J Radiat Res. 2015;56(4):727-734. doi:10.1093/jrr/rrv019. 